Proc. of the 11 Int. School on Theoretical Physics Symmetry and Structural Properties of Condensed Matter, Rzeszów 2014

\title{
Influence of Acoustic Phonons on the Magnetic Anisotropy in GaMnAs Magnetic Semiconductors
}

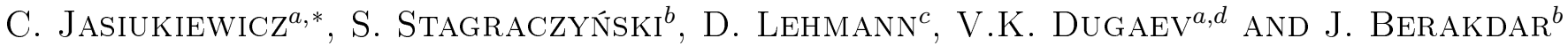 \\ ${ }^{a}$ Department of Physics, Rzeszów University of Technology, al. Powstańców Warszawy 6, 35-959 Rzeszów, Poland \\ ${ }^{b}$ Institut für Physik, Martin-Luther-Universität Halle-Wittenberg, \\ Karl-Freiherr-von-Fritsch Str. 3, 06120 Halle (Saale), Germany \\ ${ }^{c}$ Institut für Theoretische Physik, Technische Universität Dresden, Zellescher Weg 17, 01062 Dresden, Germany \\ ${ }^{d}$ Departamento de Física and CeFEMA, Instituto Superior Técnico, Universidade de Lisboa, \\ av. Rovisco Pais, 1049-001 Lisbon, Portugal
}

\begin{abstract}
We present a theoretical description of the influence of incoherent acoustic phonons on the magnetic anisotropy of magnetic semiconductors. Our theory is based on the six-band Kane model of the electron energy spectrum describing the valence band with $k \cdot p$ Hamiltonian including the hole-phonon interaction term. We include the effect of incoherent phonons through the hole self-energy in the six-band model, and assume a strong laser-pulseinduced flux of non-equilibrium acoustic phonons. The results of numerical calculations of magnetic anisotropy performed for $(\mathrm{GaMn})(\mathrm{AsP})$ magnetic alloy semiconductors demonstrate the essential role of incoherent phonons.

DOI: $10.12693 /$ APhysPolA.128.179

PACS: 75.50.Pp, 75.30.Gw, 77.80.Fm
\end{abstract}

\section{Introduction}

One of the main problems in spintronics is related to the possibility of effective control of the magnetization via some non-magnetic parameters [1]. In the literature, several examples can be found of different methods to manipulate the magnetization in ferromagnetic semiconductors without using magnetic fields [2-4]. In particular, for $(\mathrm{Ga}, \mathrm{Mn})$ As semiconductors the strong coupling between magnetic and elastic properties [3,5] allows for the strain to be a good tool to vary the magnetization direction. It is also especially important to achieve such a control on very fast time. In magnetic GaMnAs semiconductors a strong coupling between magnetic and elastic properties is evident [3].

It was reported recently in several experimental papers about an effect of the static strain in GaMnAs semiconductor [6] on the magnetic anisotropy, and a similar dynamic effect when the strain is produced by short laser pulses [7]. Also, it was demonstrated [8] a possibility to use non-equilibrium acoustic phonons generated by picosecond laser pulses for the irreversible switch of magnetization between different easy axis orientations. The effect was observed in a $100 \mathrm{~nm}$ thick film of (GaMn)(AsP) magnetic alloy semiconductor on a $350 \mu \mathrm{m} \mathrm{GaAs}(001)$ substrate in the temperature of liquid helium. The direction of magnetic easy axis in the film is determined by strain as well as shape anisotropy. In particular, a thin film of GaMnAs on a GaAs substrate exhibits a compressive strain. The concentration of phosphorus atoms was

* corresponding author; e-mail: czjas@prz.edu.pl chosen to give a small tensile strain, the effect of which is to offset the shape anisotropy, reducing the energy barrier between in-plane and out-of-plain easy axis configurations. In the experiment of Ref. [8] the short acoustic phonon pulses were generated by thermalising an intense femtosecond laser pulse in a thin metal film evaporated on the back of the substrate. The consequent rapid thermal expansion of the film launches a picosecond duration strain pulse into the substrate, followed by nanosecond duration longitudinal acoustic (LA) and transverse acoustic (TA) nonequilibrium phonon pulses, emitted as the film cools. At liquid helium temperature, all these pulses travel ballistically through the substrate until they reach the layer of interest, where they are detected.

A magnetic field $B$ was applied perpendicular to the sample plane. The sample was lithographically patterned in a low resistance Corbino disk geometry. The direction of the magnetization $M$ was inferred from measuring the Corbino resistance $R$ when a DC bias voltage was applied to it.

In order to investigate the effects of single laser pulses the magnetization $M$ was saturated along the positive direction of $B$ and then $B$ was ramped to $-0.5 \mathrm{kOe}$. At this value of $B$, the Corbino resistance $R$ was recorded while firing four single pulses, a few seconds apart from each other, followed by continuous pulses at a rate of $5 \mathrm{kHz}$. As the result of this measurement the several switching steps was observed. The first pulse was the one inducing the biggest switching step $(\Delta R / R=0.006)$, while the effect of the following ones rapidly decreased with their number.

\section{Model}

In our calculations we use the six-band Kane model [9] with effective Hamiltonian containing a standard $k \cdot p$ 
Luttinger-Kohn part [10] for the valence band with internal spin-orbit interaction, the Bir-Pikus part [11] describing the influence of a strain on the band structure, and a magnetic part for the contribution of $p-d$ exchange interaction of holes with the homogeneous magnetization [12]. We also include the interaction of holes with nonequilibrium phonon flux generated at the surface by the strong laser perturbation pulse. Then the total Hamiltonian has the following form

$$
\begin{aligned}
& \widehat{H}=\sum_{\boldsymbol{k}} \psi_{\boldsymbol{k}}^{+}\left(\widehat{H}_{0}+\widehat{H}_{m}+\widehat{H}_{\varepsilon}\right) \psi_{\boldsymbol{k}} \\
& +\sum_{\boldsymbol{k} \boldsymbol{s} \boldsymbol{q}} \psi_{\boldsymbol{k}+\boldsymbol{q}}^{+} \widehat{V}_{s \boldsymbol{q}} \psi_{\boldsymbol{k}}\left(b_{s \boldsymbol{q}}+b_{s,-\boldsymbol{q}}^{+}\right),
\end{aligned}
$$

where $\widehat{H}_{0}, \widehat{H}_{\varepsilon}$ and $\widehat{H}_{m}$ refer to unstrained GaAs, strain and magnetic parts, respectively, whereas $\widehat{V}_{s \boldsymbol{q}}$ is the matrix of phonon-hole interaction in the basis of the Hamiltonian $\widehat{H}_{0}$. The index $s$ labels the phonon branch.

The strain tensor $u_{i j}$ consists of a static part $u_{i j}^{0}$ related to the substrate deformation and the phonon-induced part $\delta u_{i j}$,

$$
u_{i j}=u_{i j}^{0}+\delta u_{i j} .
$$

In our approach $u_{i j}^{0}$ is included in $\widehat{H}_{\varepsilon}$, and $\delta u_{i j}$ is presented as the phonon field with corresponding annihilation and creation operators of phonons $b_{s \boldsymbol{q}}$ and $b_{s \boldsymbol{q}}^{\dagger}$, respectively.

The effect of non-equilibrium phonons with distribution $\delta N\left(\omega_{s \boldsymbol{q}}\right)$ on the energy spectrum of valence-band electrons can be described by the real part of electron self energy $\widehat{\Sigma}_{\boldsymbol{k}}(\varepsilon)$ due to the electron-phonon interaction. Using the standard Green function method [13] we find

$$
\begin{aligned}
& \delta \widehat{\Sigma}_{\boldsymbol{k}}(\varepsilon)= \\
& \quad 2 \sum_{n s \boldsymbol{q}} \frac{\delta N\left(\omega_{s \boldsymbol{q}}\right)\left(\varepsilon-E_{n, \boldsymbol{k}-\boldsymbol{q}}\right)}{\left(\varepsilon-E_{n, \boldsymbol{k}-\boldsymbol{q}}\right)^{2}-\left(\hbar \omega_{s \boldsymbol{q}}\right)^{2}} \widehat{V}_{s \boldsymbol{q}}^{+} \widehat{A}_{n \boldsymbol{k}-\boldsymbol{q}} \widehat{V}_{s \boldsymbol{q}},
\end{aligned}
$$

where $\widehat{A}_{n \boldsymbol{k}}$ is the spectral function of the electron Green function.

The effective Hamiltonian, which includes the self energy part

$$
\widehat{H}_{\text {eff }}=\widehat{H}_{0}+\widehat{H}_{m}+\widehat{H}_{\varepsilon}^{0}+\delta \widehat{\Sigma}_{\boldsymbol{k}}
$$

determines the dispersion of holes $E_{n \boldsymbol{k}}$ in all energy bands labeled by index $n$.

The total density of holes residing in the valence bands, $p$, is depending on the values of strain, magnetization, and on the magnetization orientation. Here we assume that the hole density is a constant determined solely by the doping with acceptors. Then we use the chemical potential $\mu$ depending on the strain and magnetization parameters.

The relation between the hole density and chemical potential $\mu$ is

$$
p(\mu)=\sum_{n \boldsymbol{k}} \theta\left[E_{n \boldsymbol{k}}-\mu\right],
$$

where $\theta(x)$ is the Heaviside step function.
The total energy of the electron system is the energy of all states which are not filled with the holes

$$
\mathcal{E}(\mu)=\sum_{n \boldsymbol{k}} E_{n \boldsymbol{k}}\left(1-\theta\left[E_{n \boldsymbol{k}}-\mu\right]\right) \text {. }
$$

This energy is depending on the direction of magnetic moment $\boldsymbol{n}$. Correspondingly, the energy of magnetic anisotropy is the difference between the energies of electron system for different magnetization orientations

$$
\delta \mathcal{E}_{a n}(p)=\mathcal{E}_{\boldsymbol{n} \| \boldsymbol{z}}(p)-\mathcal{E}_{\boldsymbol{n} \perp \boldsymbol{z}}(p),
$$

which is presented here as a function of the hole density $p$, and $\boldsymbol{z}$ denotes a direction perpendicular to the magnetic layer.

\section{Numerical results}

We performed numerical calculations of magnetic anisotropy (7) depending on the hole concentration for different values of the incoherent phonon flux and different phonon frequencies. The influence of phonons was studied separately for longitudinal (LA) and transverse (TA) phonon polarizations. Due to different propagation velocities, these two phonon modes can be easily distinguished in experiment.

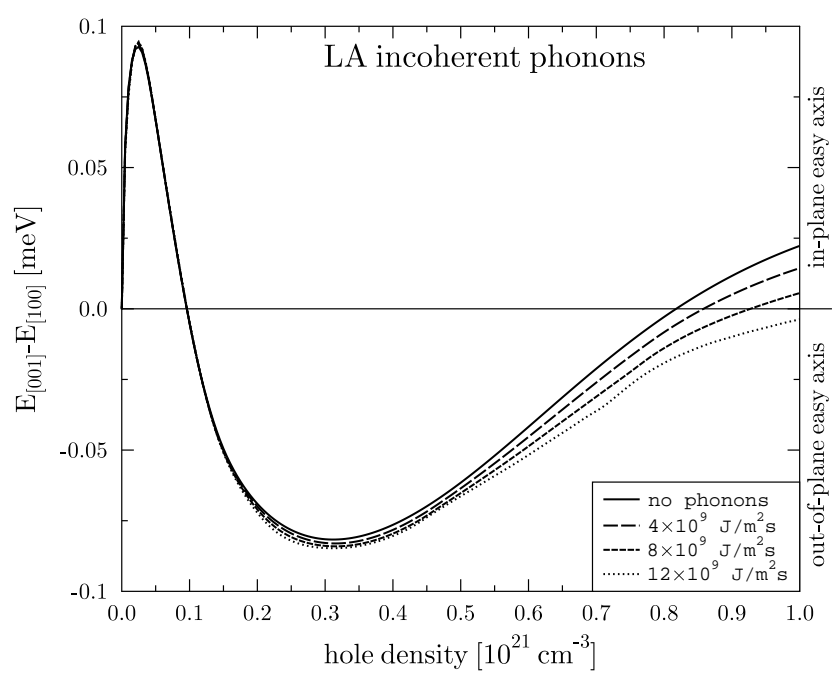

Fig. 1. Dependence of magnetic anisotropy on hole density for different values of $240 \mathrm{GHz} \mathrm{LA}$ incoherent phonon flux.

As shown in Fig. 1, the LA phonons do not affect significantly the magnetic anisotropy. This is in agreement with experimental observations of Ref. [8]. In the case of TA phonons, as shows Fig. 2, for a wide range of hole densities the value of magnetic anisotropy is strongly depending on the phonon flux. It means that the TA incoherent phonons can effectively switch the directions of magnetization easy axes from out-of-plane to the inplane configuration. This behaviour of the system was confirmed by the experiment [8]. 


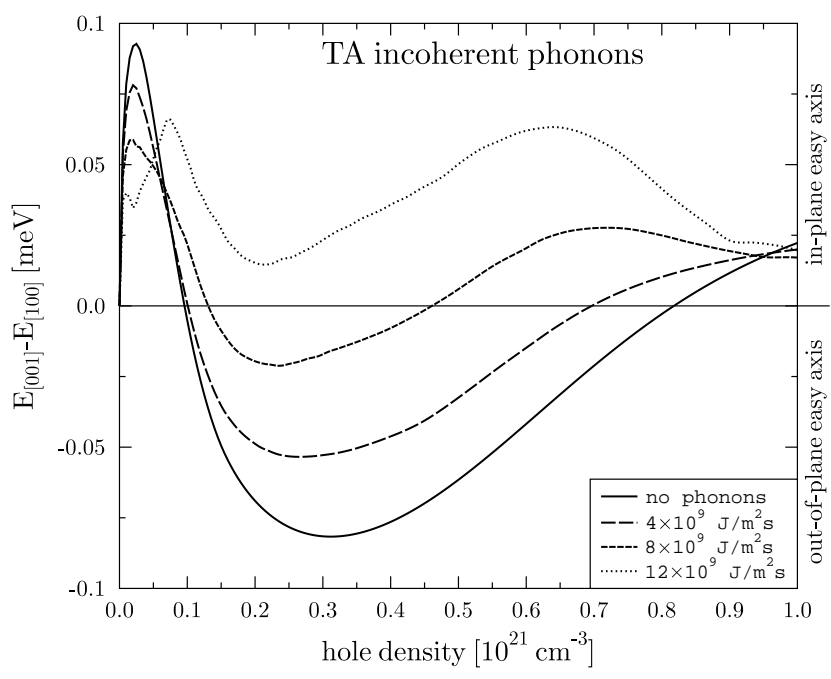

Fig. 2. Dependence of magnetic anisotropy on hole density for different values of $240 \mathrm{GHz} \mathrm{TA}$ incoherent phonon flux.

\section{Conclusions}

In this paper we demonstrate that within the six-level Kane model, it is possible to clarify the experimental results concerning using of acoustic phonon pulses to change the magnetic anisotropy in GaMnAs magnetic semiconductors. Our results are in good qualitative agreement with experimental findings.

\section{Acknowledgments}

This work is supported by the National Science Center in Poland under project Harmonia No. DEC2012/06/M/ST3/00042.

\section{References}

[1] D. Chiba, M. Yamanouchi, F. Matsukura, H. Ohno, Science 301, 943 (2003).

[2] H. Ohno, D. Chiba, F. Matsukura, T. Omiya, E. Abe, T. Dietl, Y. Ohno, K. Ohtani, Nature 408, 944 (2000).
[3] J. Wunderlich, A.C. Irvine, J. Zemen, V. Holý, A.W. Rushforth, E. De Ranieri, U. Rana, K. Výborný, J. Sinova, C.T. Foxon, R.P. Campion, D.A. Williams, B.L. Gallagher, T. Jungwirth, Phys. Rev. B 76, 054424 (2007).

[4] G.V. Astakhov, A.V. Kimel, G.M. Schott, A.A. Tsvetkov, A. Kirilyuk, D.R. Yakovlev, G. Karczewski, W. Ossau, G. Schmidt, L.W. Molenkamp, Th. Rasing, Appl. Phys. Lett. 86, 152506 (2005).

[5] J. Wenisch, C. Gould, L. Ebel, J. Storz, K. Pappert, M.J. Schmidt, C. Kumpf, G. Schmidt, K. Brunner, L.W. Molenkamp, Phys. Rev. Lett. 99, 077201 (2007).

[6] A.W. Rushforth, E. De Ranieri, J. Zemen, J. Wunderlich, K.W. Edmonds, C.S. King, E. Ahmad, R.P. Campion, C.T. Foxon, B.L. Gallagher, K. Výborný, J. Kučera, T. Jungwirth, Phys. Rev. B 78, 085314 (2008).

[7] A.V. Scherbakov, A.S. Salasyuk, A.V. Akimov, X. Liu, M. Bombeck, C. Brüggemann, D.R. Yakovlev, V.F. Sapega, J.K. Furdyna, M. Bayer, Phys. Rev. Lett. 105, 117204 (2010).

[8] A. Casiraghi, P. Walker, A.V. Akimov, K.W. Edmonds, A.W. Rusworth, E. De Ranieri, R.P. Campion, B.L. Gallagher, A.J. Kent, Appl. Phys. Lett. 99, 262503 (2011).

[9] E.O. Kane, Phys. Rev. 178, 1368 (1968).

[10] S.L. Chuang, Physics of Optoelectronic Devices, Wiley, New York 1995.

[11] G.L. Bir, G.E. Pikus, Symmetry and Strain-Induced Effects in Semiconductors, Wiley, New York 1974.

[12] T. Dietl, H. Ohno, F. Matsukura, Phys. Rev. B 63, 195205 (2001).

[13] A.A. Abrikosov, L.P. Gorkov, I.E. Dzyaloshinski, Methods of Quantum Field Theory in Statistical Physics, Dover, New York 1963. 